\title{
JAPANESE ANIMAL TOURISM AND THE KAWAII (CUTE) AESTHETIC
}

\author{
Émilie Crossley
}

\section{INTRODUCTION}

Japan is rapidly developing a global reputation as a tourism hot-spot for animal lovers. Opportunities for tourists to interact with animals can be found across a range of settings throughout the country, from the famous snow monkeys of Nagano, to free-ranging deer in Nara, to the assortment of cat, and other animal cafés in Tokyo. Many such attractions are marketed as a chance to experience animals that are described as "cute", "adorable" or "fluffy". These portrayals draw on the "kawaii" aesthetic, which is a prominent feature of Japanese popular culture. Kawaii is a culturally specific form of cuteness that often references animal characters or animal-like qualities. While sociological explorations of the cultural phenomenon abound, the implications of characterising real animals as kawaii, such as those used in the tourism industry, have received limited academic attention. However, recently the welfare of Japan's cute animals has come under scrutiny from international tourists, sparking vocal criticism on social media and even petitions calling for unethical attractions to be closed down. I explore how kawaii has shaped the terrain of contemporary Japanese animal tourism and how contestation of the aesthetic is opening up debate regarding animal welfare and ethics in Japan.

\section{KAWAII:THE CULT OF CUTE}

The kawaii, or "cute", aesthetic has become an integral part of Japanese popular culture. Kawaii is a ubiquitous term used to describe a range of cultural artefacts, including fictional characters such as Hello Kitty or Pikachu, animal-like regional mascots known as "yuru-kyara", bento boxes containing rice balls shaped to look like characters or animals known as "kyaraben", and, of course, cat cafés. The term is also commonly used in reference to a hyper-feminine, girlish fashion style, which often features pink babydoll dresses, knee-high socks, frills and bows reminiscent of children's clothes, and animal ear headbands. While the domestic appeal of kawaii cannot be denied, the aesthetic has also proven to be a valuable, exportable commodity as part of the national "Cool Japan" brand and ideology.' Figures such as Hello Kitty have become internationally recognisable icons of Japanese popular culture. ${ }^{2}$ So successful has the globalisation of kawaii been that many people outside of Japan will intuitively recognise this version of cuteness, with its pastel colours, childlike innocence and anthropomorphised animals, even if they have never heard of its Japanese name.

While kawaii translates as "cute" in English, the Japanese term has a broader semantic meaning. Kawaii is derived from "kawahayushi", which is used to describe someone blushing in embarrassment ${ }^{3}$ and the word also shares an etymological link with "kawaisō", meaning pitiable or pathetic. ${ }^{4}$ Thus, while the Anglophone and Japanese terms both bring to mind smallness, youth and innocence, kawaii carries additional connotations of embarrassment, obedience, weakness and dependency. There is also a nostalgic element to kawaii, invoking memories of childhood warmth, sweetness and comfort. ${ }^{5}$ Kawaii, or cuteness, is often conceptualised relationally rather than as a property belonging to an object or person; it is understood as a "performative act expressing affinity"6 or a "mode of regard"7 that establishes a relationship of empathy, care and intimacy. Parkinson emphasises the power dynamic inherent in such relationality, 
suggesting that cuteness relies on inequality between "the diminished weaker object and the larger stronger subject."' While kawaii provides an outlet for the desire to nurture, for some the desire is to be the one who is nurtured. This relates to the Japanese concept of "amae", which denotes a childish desire to be indulged, taken care of and loved."

As a cultural phenomenon, kawaii emerged in the 1970s, following a period of social and political unrest. One of the first expressions of kawaii to be noticed was in the handwriting of female high school students in Japan, who began using childish, rounded letters and characters, and punctuating their text with little drawings. ${ }^{10}$ In response to the prevailing cultural norms of seriousness, rigidity and responsibility, these girls mounted a "graphological rebellion" that used cuteness to challenge adult authority." Other appraisals suggest that a love of cuteness is more deeply rooted in Japanese culture and can be traced back to the Edo Period when miniature carved "netsuke" were popular collectable items. ${ }^{12}$ Equally, some credit the artist Rune Naito as a pioneer in the aesthetic of kawaii through his magazine illustrations of baby-faced ("nitōshin") girls in the 1950s. ${ }^{13}$ Regardless of its origins, kawaii has faced a domestic backlash, particularly from feminists who perceive the aesthetic as fetishizing and disempowering girls. ${ }^{14}$ Outside of Japan, the reception has been mixed and, while many have embraced and emulated the kawaii aesthetic, it has also been labelled an "obsession" that has promoted a "cult of cute."'15

\section{KAWAII ANIMALS IN JAPAN}

Animality is a prominent feature of kawaii, and Masabuchi lists "animal-like qualities" as one of the seven elements that define cute in the Japanese context. ${ }^{16}$ Stylised depictions of animals and animal characters appear on consumer products such as clothes, accessories, toys, games and food. Animal-like mascots known as "yuru-kyara" have been used as a city branding strategy in an effort to increase tourist visitation, and each year Japan hosts a Grand Prix to find the country's most popular mascot. ${ }^{17}$ One of the most iconic yuru-kyara is Kumamon, the mascot of the Kumamoto prefecture. Kumamon has the appearance of an anthropomorphic bear with rosy red cheeks, a wide smile, and a perpetual look of surprise on his face. While Kumamon is instantly recognisable as an ursine mascot, the official word from the Kumamoto Prefecture is that he is in fact not a bear, although we do not learn what sort of creature he is instead. ${ }^{18}$ Similarly, many are surprised to learn that Hello Kitty is actually a little girl rather than the cat she resembles. ${ }^{19}$ Kawaii representations are thus often ambiguous, leaving the viewer uncertain whether they are encountering an anthropomorphised animal or an animalesque human.

Animals as cute characters and mascots have been subject to research, particularly given the recent rise of "contents tourism" - a term originating in Japan that denotes travel motivated by narratives, characters, and other aspects of popular culture..$^{20}$ However, what has been left relatively unexplored is how Japan's obsession with cuteness impacts the lives of real animals. A lucrative industry has emerged to cater for the desires of those who choose to have "fur babies" rather than human babies"1; a preference that has been problematic for Japan given its declining birth rate and aging population. Instead of procreating, many Japanese people choose to shower affection on their hugely indulged pets. The sight of small dogs dressed in cute clothes being pushed around the streets in strollers, just like babies, is not uncommon. This expression of kawaii is arguably benign, at least in terms of the effect on the companion animals. However, more ethically questionable practices can be found in the tourism and hospitality industries, where animals are put on display for the benefit of paying customers. Tourism blogs compiling lists of the "top five natural kawaii places to visit in Japan"22 or the "cutest things to do in Tokyo"23 often direct prospective tourists to animal attractions. The power of animals as tangible embodiments of kawaii is clear and, as one blogger puts it, "Nothing screams kawaii more than places filled with loveable furry animals." 24

A prominent example of kawaii used in animal attractions is the phenomenon of cat cafés, which emerged initially for the domestic market as part of the "iyashi" (healing) boom that followed the economic recession of the 1990s. Particularly fashionable in Tokyo, cat cafés provide relaxing, intimate spaces that allow urbanites to get away from the stresses of everyday life and to experience an aesthetic of domesticity that has become unattainable to many. ${ }^{25}$ In this context, feline cuteness is framed as therapeutic and the cats' softness and carefree demeanour are echoed 
in the atmosphere that the cafés actively seek to cultivate. The iyashi qualities of cats include "their physical warmth and the visceral, pleasurable texture of their fur."26 Plourde notes that cat cafés are often decorated in ways that are "childlike, innocent, and non-threatening", using pastel colours, round shapes, soft furniture and cosy blankets - all elements that are synergistic with the kawaii aesthetic. While Japan's cat cafés reached the peak of their popularity in 2009, the craze sparked a new generation of animal cafés. In addition to cat cafés, one can now visit cafés featuring dogs, capybaras, piglets, goats, hedgehogs, owls, rabbits, otters, chinchillas, fennec foxes, parrots, hamsters and flying squirrels. While data on this new wave of animal cafés are limited, it seems likely that international tourists are at least one intended target market.

HARRY - a hedgehog café in Tokyo - invites customers to enjoy a cup of coffee and to "play with cute animals" that are "soft, warm, and at times cheeky."27 HARRY's website is adorned with stylised illustrations of little smiling hedgehogs and a photograph of two hedgehogs on the homepage is overlaid by the words "so cute!" that appear repeatedly over their heads. These hedgehogs are set against a pastel pink background, channelling the kawaii aesthetic. HARRY is listed as the number one kawaii thing to do in Tokyo, according to an online list published this year. ${ }^{28}$ The hedgehogs can be observed in glass tanks, fed mealworms and handled using special gloves to protect customers against their spines as, despite the website's suggestion, hedgehogs are not all that soft. Customers enthusiastically document their encounters with the hedgehogs, reporting "cuteness overload" at the "overwhelmingly adorable" creatures. ${ }^{29}$ Common features that are identified as cute include their small size, little pink snouts, round ears, yawning and sleepiness. One blogger reports that she "just about melted inside" when a hedgehog curled up in a ball and fell asleep in her hands, adding that a photograph of her partner holding the hedgehog "looked like he was a proud father, holding his new-born baby for the very first time." ${ }^{30}$

In a very different setting, Zao Fox Village in Nagano Prefecture is advertised as being home to "I 00 fluffy cute foxes" ${ }^{\prime \prime 1}$ and foreign travel bloggers have enthusiastically described the attraction as the "cutest" 32 and "fluffiest" 33 place on Earth. The "village" can be classified as an animal park, or small niche zoo, that allows the foxes to roam freely in its woodland setting. Some foxes, however, are kept in cages and cubs are available to cuddle at certain times of the year. Its website features a smiling, winking yellow fox in the logo, images of recently born cubs, and a rather comical photograph of a fox wearing a train conductor's hat during its inauguration as a stationmaster for Shiroishizaō Station. The kawaii aesthetic presents differently in relation to foxes compared with hedgehogs; it is not so much their small stature that visitors adore as their "fluffy mischievousness". ${ }^{34}$ The foxes are reported as being a bit naughty with a tendency to nibble at tourists' clothes and bags. However, given that visitors are prohibited from touching the foxes, this mischief is often seen as a welcome interaction and all part of the animals' charm. Many tourists recommend visiting the village in winter when the foxes' coats are thickest and fluffiest; the snow providing the perfect backdrop for their bright fulvous fur and endowing the park with a magical, fairy-tale quality. ${ }^{35}$ Similar to the hedgehogs, when the foxes are curled up asleep they form that familiar soft, round, chubby shape that is suggestive of kawaii.

Use of the kawaii aesthetic in the branding, marketing and physical setup of Japanese animal attractions has implications for how tourists perceive and experience these sites. On the simplest level, the kawaii label can act as a drawcard that entices tourists to visit animal attractions, ensuring that businesses will be listed on "top ten" lists of cute things to do in Japan and reframing animals as a cultural tourism experience. It is also possible that kawaii, as a superimposed frame of reference, affects how tourists see the animals at these attractions, heightening their awareness of certain physical or behavioural characteristics such as smallness, fluffiness, softness, roundness, sleepiness and cheekiness. Equally, the atmosphere of childlike innocence that the kawaii aesthetic provides, primes tourists for a benign, guiltless experience. Positive visual cues can be found everywhere, from the smiling cartoon hedgehogs and foxes depicted on the HARRY and Zao FoxVillage websites to these same characters that welcome visitors onsite, emblazoned on signs, all of which is designed to provide reassurance and comfort. In addition, animal behaviours that might be found problematic in another context find an explanation in kawaii. Hedgehogs yawn not because they are exhausted from constant daytime handling, but simply because they are being cute. Foxes bite at the bags of visitors not out of boredom or because they are hungrily searching for food, but because they are expressing their cute mischievousness. 


\section{ANIMAL AESTHETICS AND ETHICS}

Kito argues that Japan's understanding of animal ethics and treatment of animals in the tourism industry are problematic, going so far as to liken some Japanese animal attractions to a form of "dark tourism". ${ }^{36}$ This (mis) treatment of animals can be related to a pervasive anthropocentrism that infuses the tourism industry globally and to which Japan is not immune. ${ }^{37}$ The animal attractions reviewed in this paper have proven to be polarising, with not all visitors gleefully experiencing "cuteness overload". A cursory glance at tourism review websites such as TripAdvisor reveals tourists' perceptions of the "unethical" or even "cruel" treatment of the animals. In the case of HARRY, tourists express concern about keeping the nocturnal hedgehogs awake during the day, inadequate supervision of customers to ensure that the hedgehogs are being correctly handled, a lack of enrichment in their glass tanks, and signs that the hedgehogs are stressed, exhausted and depressed. In the case of Zao Fox Village, concerns include foxes being kept in cages, sedation of foxes for petting, fighting resulting from overcrowding and competition over food, poor health including open wounds and malnourishment, and disturbance of sleeping foxes (which are also naturally nocturnal). Indeed, the conditions at Zao FoxVillage have caused such consternation that an online petition has been set up calling for the facility to be closed down. ${ }^{38}$

Mkono and Holder conceive of social media as digital spaces of collective moral reflexivity, which signal heightened public engagement in human-animal recreational ethics. ${ }^{39}$ Meaning and morality, in relation to tourist experience, are routinely engaged with, co-created and contested through online forums. Kawaii has thus become embroiled in online debate regarding animal welfare and ethics. In the case of Zao Fox Village, enthusiastic blogs and vlogs branding the facility as the "cutest" and "fluffiest" place on Earth have been rebuked by online responses exposing why, to quote one author, it is "not the cutest place on Earth". 40 In the comments of this particular blog entry, the word "cute" appears frequently between inverted commas, hinting at suspicions that the term has come to be used deceptively, concealing a dark reality. For some, kawaii has been revealed as a marketing ploy and product of media hype. However, not everyone agrees that cruelty exists behind the smiling face of the cartoon animals beckoning us to visit these attractions. Satisfied customers argue that the appearance or behaviour of animals that can appear disturbing is often perfectly natural. Patchy fur, taken as a sign of poor health, can result from shedding in the summer to keep the foxes cool. Fighting over food is a normal way for foxes to assert their dominance. And while foxes and hedgehogs are indeed nocturnal in the wild, it is possible for domesticated animals to adapt to new routines, much like house cats who are naturally crepuscular (active mainly at dawn and dusk).

The intersection between the kawaii aesthetic and animal ethics is complex. It is widely accepted that the animals we find cute display morphological neoteny: juvenile physical traits such as large eyes, a big head, round body, short limbs and soft textures. ${ }^{41}$ These features, also referred to as the "kindchenschema" (baby schema) ${ }^{42}$, remind us of human babies and trigger the cute response. We are particularly drawn to mammalian neoteny and baby mammals, such as puppies and kittens, represent the height of cute. Estren argues that neoteny presents a barrier in animal ethics, making us more inclined to divert research and conservation efforts towards cute animals at the expense of the "non-cute". ${ }^{43}$ However, even for those animals designated as cute, relationships with humans can be problematic. May argues that the anthropomorphism of cute presents an "imperial force that brings everything under human sway", resisting any acceptance or understanding of Otherness. ${ }^{44}$ However, he also suggests that anthropomorphism can be conceptualised more favourably as an attempt to humanize the Other and extend our circle of moral concern and empathy. It is this tension that plays out in Japanese animal attractions; tourists are called upon by kawaii to adore animals, yet in the process they risk shielding themselves from the inherent unknowability of another species. While the desire to care for animals may be admirable, without a solid knowledge base about them we may inadvertently cause harm. Finally, Parkinson reminds us that cuteness "invites care through the affective draw of inequalities of power". ${ }^{5}$ Is such a relation of care, premised upon the belief that animals are weak and in need of human protection, ethical or desirable? 


\section{CONCLUSION}

In this paper, I have shown how the popular kawaii aesthetic has implications for animals used in Japan's tourism industry. Cute sells, and Japan's quirky brand of cute has proven to be particularly lucrative. While previous research has explored how kawaii practices shape anthropomorphic animal characters, mascots and merchandise, relatively little attention has been devoted to how real animals are affected by their designation as cute. I have identified how the kawaii aesthetic is used to market animal attractions in Japan and to disarm tourists with their façade of innocence. Tourists trying to make sense of their experiences engage in collective moral reflexivity via social media, which has drawn the terms "kawaiil" and "cute" into contention. Some report the condition or behaviour of animals that, while at first appearing to be cute, indicate potential animal neglect and cruelty. The anthropomorphising tendency of kawaii reveals an ethical quandary, both engendering empathy with other species and risking harm by resisting an understanding of animal Otherness in all its wondrous complexity. It is this potential for care and for harm that must be weighed up when gauging the ethical value of Japanese animal attractions.

What the future holds for Japan's animal tourism industry depends both on customer demand and government regulations. Mindful of the rise in scrutiny and criticism that the sector has faced in recent years, some animal tourism businesses are adapting. For example, the cat café franchise, Neco Republic, is now working with animal shelters in an effort to rehouse stray cats through their cafés. ${ }^{46}$ Increasingly, travel blogs and tourism websites are including statements regarding the ethical status of particular animal attractions, encouraging consumers to be more discerning. Also, as I have indicated, tourists are beginning to question naturalised labels such as "kawaiil" and "cute" that are used to promote animal tourism. We are currently at a pivotal moment in the history of global tourism; disruption caused by the COVID-19 pandemic is leading many scholars, practitioners and activists to re-envision what tourism may look like in the future. While there will be immense pressure to rebuild a viable industry post-pandemic, there is a chance, and perhaps a desire, to rebuild with sustainability, ethics and the welfare of animals in mind. ${ }^{47}$

Dr Émilie Crossley is a tourism researcher whose work draws on social psychoanalytic theory to approach the psychological in tourism in a non-reductive, culturally engaged way. Her research explores tourist subjectivity, cosmopolitan empathy, and reflexivity. Émilie's research has been published in international journals including Tourism Geographies and Tourism Recreation Research.

I Laura Miller, "Cute Masquerade and the Pimping of Japan," International Journal of Japanese Sociology 20, no. I (20 I I): I 8-29.

2 Christine R. Yano, Pink Globalization: Hello Kitty's Trek Across the Pacific (London: Duke University Press, 20I 3).

3 Toby Slade, "Cute Fashion: The Social Strategies and Aesthetics of Kawaii," in Introducing Japanese Popular Culture, eds. Alisa Freedman and Toby Slade (Abingdon: Routledge, 2018), 399-4II.

4 Yano, Pink Globalization.

5 Ali Soltani, Johannes Pieters, Janette Young and Zhaohong Sun, "Exploring City Branding Strategies and Their Impacts on Local Tourism Success, the Case Study of Kumamoto Prefecture, Japan," Asia Pacific Journal of Tourism Research 23, no. 2 (2018): 158-169.

6 Joshua P Dale, “The Appeal of the Cute Object: Desire, Domestication, and Agency," in The Aesthetics and Affects of Cuteness, eds. Joshua P Dale, Joyce Goggin, Julia Leyda, Anthony P Mclntyre and Diane Negra (Abingdon: Routledge, 20I6), 45-65. 
Yano, Pink Globalization, 57.

8 Claire Parkinson, Animals, Anthropomorphism and Mediated Encounters (Abingdon: Routledge, 2019).

9 Caroline S.L.Tan, “ご当地キャラ (Gotochikyara) \& ゆるキャラ (Yurukyara)-The Fusion of Pop Culture in Place Branding in Japan," UTCC International Journal of Business and Economics 6, no. I (20।4): 73-89.

10 Sharon Kinsella, "Cuties in Japan" in Women, Media and Consumption in Japan, eds. Lise Skov and Brian Moeran (Richmond: Curzon Press, 1995), 220-254.

I I Slade, "Cute Fashion."

12 "Japan Smitten by Love of Cute," The Age, https://www.theage.com.au/entertainment/celebrity/japan-smitten-by-love-of-cute200606 19-ge2jna.html (accessed April 26, 2020).

13 Rune Naito, "Roots of Kawaii," https://www.naitou-rune.jp/ (accessed April 26, 2020).

14 Miller, "Cute Masquerade."

15 Hui-Ying Kerr, "What is Kawaii - and Why Did the World Fall For the 'Cult of Cute'?" The Conversation, https://theconversation. com/what-is-kawail-and-why-did-the-world-fall-for-the-cult-of-cute-67I 87 (accessed April 26, 2020).

16 Sōichi Masabuchi, Kawaii Shokogun (Tokyo: NHK Publishing, 1994).

17 Soltani et al., "Exploring City Branding Strategies."

I8 Debra J. Occhi, "Kumamon: Japan's Surprisingly Cheeky Mascot," in Introducing Japanese Popular Culture, eds. Alisa Freedman and Toby Slade (Abingdon: Routledge, 2018), I3-23.

19 Alexandra Petri, "Hello Kitty is Not a Cat. Everything is a Lie," The Washington Post, https://www.washingtonpost.com/blogs/ compost/wp/2014/08/27/hello-kitty-is-not-a-cat-everything-is-a-lie/ (accessed April 26, 2020).

20 Takayoshi Yamamura and Philip Seaton, eds. Contents Tourism and Pop Culture Fandom:Transnational Tourist Experiences (Bristol: Channel View Publications, 2020).

21 Ruth Evans and Roland Buerk, "Why Japan Prefers Pets to Parenthood," The Guardian, https://www.theguardian.com/ lifeandstyle/2012/jun/08/why-japan-prefers-pets-to-parenthood (accessed April 26, 2020).

22 "Top 5 Natural Kawaii Places in Japan," Klook, https://www.klook.com/blog/top-5-natural-kawaii-places-in-japan/ (accessed April 26, 2020).

23 B.M. Naho, "I 0 Kawaii Things to Do in Tokyo," Japan Wireless, https://jw-webmagazine.com/l 0-cutest-things-to-do-in-tokyo2018-75 I6bfb28a76/ (accessed April 26, 2020).

24 "Top 5 Natural Kawaii," Klook.

25 Lorraine Plourde, "Cat Cafés, Affective Labor, and the Healing Boom in Japan,'Japanese Studies 34, no. 2 (20 I 4): I I5-133.

26 Ibid, 120.

27 Hedgehog Cafe HARRY. Accessed April 27, 2020. https://www.harinezumi-cafe.com/en/

28 Naho, "I0 Kawaii Things."

29 B M Naho, "Have the Most Unique and Kawaii Experience at the Hedgehog Café HARRY in Roppongi, Tokyo!" Japan Wireless, https://jw-webmagazine.com/cuteness-overload-hedgehog-cafe-harry-in-roppongi-tokyo-955c3687673/ (accessed April 26, 2020).

30 Lauren Juliff, "Should You go to a hedgehog café? My experience in Japan," Never Ending Footsteps, https://www. neverendingfootsteps.com/harry-hedgehog-cafe-japan/ (accessed April 27, 2020).

31 Zao Fox Village, http://zao-fox-village.com/en (accessed March 27, 2020).

32 Julija Neje, "FoxVillage in Japan is Probably the Cutest Place on Earth," Bored Panda, https://www.boredpanda.com/zao-foxvillage-japan/ (accessed April 27, 2020).

33 Aileen Adalid, "Fluffiest Place on Earth: Miyagi Zao Fox Village (Japan)," I Am Aileen, https:/liamaileen.com/fox-village-japancute-kitsune-how-to-travel-guide/ (accessed April 27, 2020).

34 Neje, "FoxVillage in Japan."

35 "Zao FoxVillage," GajinPot Travel, https://travel.gaijinpot.com/zao-fox-village/ (accessed April 26, 2020).

36 Yoko Kito, "The Metaphysical Background of Animal Ethics and Tourism in Japan," in Tourism Experiences and Animal Consumption, ed. Carol Kline (Abingdon: Routledge, 2018), 165-178.

37 Atsuko Hashimoto, "Environmental Perception and Sense of Responsibility of the Tourism Industry in Mainland China, Taiwan and Japan," Journal of Sustainable Tourism 8, no. 2 (2000): | $31-146$.

38 "Tell Japan to Shut Down Zao Fox Village!" Change.org, https://www.change.org/p/prime-minister-of-japan-shut-down-zaofox-village (accessed March 16, 2020). 
39 Mucha Mkono and Afiya Holder, "The Future of Animals in Tourism Recreation: Social Media as Spaces of Collective Moral Reflexivity," Tourism Management Perspectives 29 (2019): I-8.

40 Jessica Van Roij, "Why Zao Fox Village is Not the Cutest Place on Earth," Jessikatrips, http://jessikatrips.com/why-zao-foxvillage-is-not-the-cutest-place-on-earth/ (accessed April 27, 2020).

4I Mark J Estren, "The Neoteny Barrier: Seeking Respect for the Non-cute,"Journal of Animal Ethics 2, no. I (2012): 6-1।.

42 Maura L Scott and Gergana Y. Nenkov, “Using Consumer Responsibility Reminders to Reduce Cuteness-Induced Indulgent Consumption,"'Marketing Letters 27, no. 2 (2016): 323-336.

43 Estren, "The Neoteny Barrier."

44 Simon May, The Power of Cute (Princeton: Princeton University Press, 2019), I 10.

45 Parkinson, Animals, Anthropomorphism.

46 Kaori Shoji, "Cat Cafe Operators in Japan Seek to End Stigma with a Fresh Approach," The Japan Times, https://www. japantimes.co.jp/news/2019/1 2/2 I/national/media-national/cat-cafe-operators-japan-seek-end-stigma-fresh-approach/\#. Xqf9QNMzZZO (accessed April 28, 2020).

47 Émilie Crossley, "Ecological Grief Generates Desire for Environmental Healing in Tourism After COVID-19," Tourism Geographies (Advance online publication, 2020). https://doi.org/ | 0.1080/|46 | 6688.2020.1759|33 